\title{
The Relation Between The Soil Taxonomic Units Developed on The Geomorphic Units and Soil Fertility Status in The West Delta, Egypt.
}

K. S. El-Hedek, I. A. Hegab, E. E. Massoud and G. Farid

Soil, Water and Environment Research Institute, Egypt.

7 LEVEN soil profiles represent the different geomorphic units in d west Delta were chosen to study the relation between the soil taxonomic units developed on the geomorphic units and soil fertility status. The obtained results indicated that the taxonomic units are: 1) Typic Haplocalcids , Typic Calcigypsids developed on old Alluvial soils 2) Typic Torripsamment developed on windblown sand 3) Aquic Haplocalcids developed on Fluvio-Lacustrine soils . 4) Typic Torrifluvents and Typic Haplotorrerts developed on recent Nile Alluvial soils. 5) Halic Endoaquerts and Aridic Endoaquerts developed on Fluvio-marine soils and 6) Oxyaquic Torripsamments developed on coastal plain soils. The organic matter contents were relatively low ranged from 0.15 to $2.35 \%$. The cation exchange capacity ranged from 2.3 to $38.3 \mathrm{meq} / 100 \mathrm{~g}$, the lowest values were found in the windblown sand, whereas, the highest were in the clayey soils of Fluvio-marine.

The total contents of iron ranged from 0.2 to $1.2 \%$, whereas the available contents ranged from 2.4 to $57.6 \mathrm{ppm}$. The independent factors of both clay, silt and $\mathrm{CaCO}_{3}$ contributed 37.1 to 89.7 of the variations in iron in the different studied soils. The total manganese contents ranged from 170.0 to $1048.0 \mathrm{ppm}$, whereas the available contents ranged from 0.48 to $5.28 \mathrm{ppm}$. The independent factors of both clay, silt and $\mathrm{CaCO}_{3}$ contributed 22.7 to $93.4 \%$ from the variations in $\mathrm{Mn}$ contents in those studied soils. The total contents of $\mathrm{Zn}$ ranged from 47.0 to $370.0 \mathrm{ppm}$, whereas, the available contents ranged from 0.48 to $5.28 \mathrm{ppm}$. The independent factors of clay, silt and $\mathrm{CaCO}_{3}$ were responsible 49.3 to $81.0 \%$. The total copper contents ranged from 22.0 to $168.0 \mathrm{ppm}$, whereas the available content ranged from 0.89 to $9.9 \mathrm{ppm}$. The variations of total copper were related to the independent factors of both clay, silt and $\mathrm{CaCO}_{3}$, by 10.9 to $90.0 \%$.

The Taxonomic unit of Typic Haplotorrerts and Endaquerts developed on the flood plain and Fluvio-marine soils was the highest fertile status in the studied area, whereas the Typic Torripsamments were the lowest fertile status.

The fertility of the soil maybe assessed by means of the potential fertility and the actual fertility. The former concerns the weathering stage of the soil material, the base saturation and exchangeable ions adsorbed at the soil's complex and the 
quality and quantity of organic matter. It expressed by the $\mathrm{pH}$, the CEC, exchangeable cations and available $\mathrm{P}$ and C\% (FAO, 1976)

The information of concentration of the trace elements such as iron, manganese, zinc, cupper and cadmium in the genetic horizon may be useful in estimating the degree of leaching and weathering in soil profile (El-Demerdashe, 1970). The contents of $\mathrm{Zn}$ in soils depend on the parent materials, the content of organic matter, soil texture and soil $\mathrm{pH}$. He also indicated that the alluvial soils of west Delta had a high content of both boron, manganese, copper cubolat and zinc followed by the Fluvio marine soils, then the Fluvio- lacustrine.

McKenzie (1959) concluded that the concentrations of $\mathrm{Cu}, \mathrm{Zn}$ and $\mathrm{Mn}$ are releated to the difference of soil parent materials.

Oertel and Giles (1967) showed that the concentration of trace elements varies with depth in soil profile and differs from element to element depending on the morphology and Taxonomic unit of profile.

The soils derived from clay deposits were rich in manganese contents (Vinogradov, 1959). He added that the concentration of copper in soil profiles depended on the nature of the parent materials, Fiskell (1965) indicated that $\mathrm{Cu}$ is found in soils as exchangeable ions bound in organic compounds and in the crystal lattice of the aluminosillicates and other mineral.

In his study on the geomorphic features in west Delta Badawi (1999) reported that the contents of both iron, manganese, zinc and copper were: 1.56 , $0.34,0.13,0.19$ to $6.23,1.34,168,0.62 \mathrm{ppm}$ in the river terraces and $1.86,0.97$, $0.63,0.34$ to $1.89,1.02,0.68,0.65 \mathrm{ppm}$ in the windblown soils.

The aim of this investigation is studying the relation between the fertility status of the soil taxonomic units developed on different geomorphic units in west Delta.

\section{Geomorphic aspects}

The studied area is located in western Delta, Egypt between latitude of $30^{\circ}$ $21^{\prime}$ to $31^{\circ} 25^{\prime}$ north and longitude of $30^{\circ} 00^{\prime}$ to $30^{\circ} 45^{\prime}$ east and boarded by coastal sea in the north, Rosetta branch in the east, Cairo-Alexandria desert road in the west and considered as one of the most promising areas to increase the Egyptian economic.

According to Al-Sharif et al. (2013), this area contains the following geomorphic units: coast plain in the north, levee in the east on the side of Rosetta branch, flood plain in the middle, old alluvial in the south, Fluvio lacustrine in the west north, fluvio marine and windblown as illustrated in Map (1).

\section{Geology}

The recent alluvial deposits of both flood plain and levee were deposited from the suspended matter of the Nile river on the Holocene age (Ball, 1939). 
Fluvio lacustrine and fluvio marine contain two different geologic materials affected by the sea deposits in sub soil and the Nile deposits in surface mixed together forming lithological discontinuity in theses soils (El-Demerdashe, 1970).

The coastal plain was formed by the marine deposits on the Pliocene age.

The old alluvial soil in this study is considered the oldest terrace in Egypt deposits on the Pliocene age and covered by gravel pavement (Sandford and Arkell, 1939) in some places, the windblown sand covers this old alluvial.

\section{Materials and Methods}

Eleven soil profiles were chosen to represent the different geomorphic units in the studied area : The old alluvial soil (profile No. 1\&2), the windblown sand (profile No. 3), the fluvio lacustrine (profile No $4 \& 5$ ), the flood plain (profile No. 6 \& 7), the levee (profile No. 8), Fluvio-marine (profile No $9 \& 10$ ) and the coastal plain (profile No. 11). These profiles were dug to $150 \mathrm{~cm}$ from soil surface, described in the field according to FAO (2010), the soil color was performed using Munsell chart (1975). Particle size distribution was determined according to Page et al. (1982). The texture classification was performed using the American triangle chart. Soil $\mathrm{pH}$ was determined in soil paste, soluble ions, electrical conductivity (ECe) of soil paste extract calcium carbonate and gypsum contents were determined according to Page et al. (1982). Soil taxonomy was performed according to Soil Survey Staff (2010).

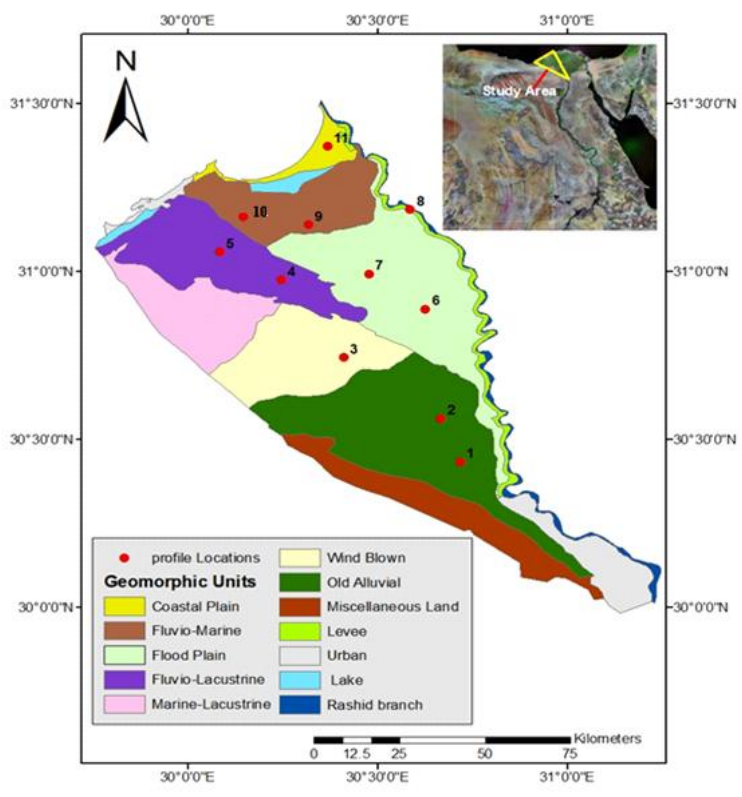

Map. 1. Geomorphic units and profiles location of the Western Delta, Egypt.

Egypt. J. Soil. Sci. 55, No. 1 (2015) 
Available trace elements were extracted by the DTPA (diethylene triamine penta acetic acid) according to the method of Lindsay and Norvell (1978).

Aliquots of 0.5 to $2.0 \mathrm{~g}$ of dried soil samples were digested in $100 \mathrm{ml}$ micro kjeldahl flasks containing $20 \mathrm{ml}$ of a mixture of concentrated $\mathrm{HNO}_{3}, \mathrm{HClO}_{4}$ (3: 1: 1) according to Grosuch (1959). The digestate was cooled and diluted to $100 \mathrm{ml}$ with deionized water.

The trace elements of $\mathrm{Fe}, \mathrm{Mn}, \mathrm{Zn}$ and $\mathrm{Cu}$ were determined using a Pye Unicum sp 1900 atomic adsorption spectrophotometer.

\section{Results and Discussion}

\section{Organic matter content}

The contents of organic matter in the studied area ranged from 0.15 to $2.35 \%$ as shown in Table 1. The highest values were found in the surface horizon (Ap) of the soils developed on Fluvio-Lacustrine, Recent alluvial and Fluvio- marine and ranged from 1.67 to $2.35 \%$ related to the added organic fertilizer. Also, these contents of organic matter decreased with soil depth except in studied profile No. 1 of the old alluvial soil due to the occurrence of the lithological discontinuity between its horizons caused by the different depositional environmental. The lowest contents were not more than $0.17 \%$ in the soils developed on windblown sand soils No. 3 due to their sandy nature.

\section{Cation exchange capacity and exchangeable cations}

Cation exchange capacity of the studied soils ranged from 2.30 to 38.3 meq/100 g. The lowest values were 2.3 and $5.4 \mathrm{meq} / 100 \mathrm{~g}$, respectively in the windblown sandy soils, and coastal plain whereas the highest values were 38.3 $\mathrm{meq} / 100 \mathrm{~g}$ as shown in the Fluvio-marine soils (Table 1).

Both the studied soils of windblown sand and coastal plain (soil profile No's $3 \&$ 11) had the lowest values of CEC lower than $5.4 \mathrm{meq} / 100 \mathrm{~g}$. This is related to their nature sandy deposits reached to more than $95.0 \%$ sand and also to their low contents of organic matter, lower than $0.5 \%$. The highest values were in the Fluvio-marine soils (profile No. $9 \& 10$ ) reached to $38.3 \mathrm{meq} / 100 \mathrm{~g}$ and flood plain reached to $38.0 \mathrm{meq} / 100 \mathrm{~g}$ due to their clayey texture whereas clay contents reached to $60.0 \%$ and relatively high contents of organic matter in the surface horizons. These soils followed by the studied soils of both Fluvio-lacustrine and Levee (soil profile No. 4, 5 and 8) which had moderate values of CEC ranged from 14.3 to $28.8 \mathrm{meq} / 100 \mathrm{~g}$ due to their medium texture; i.e. clay loam to sandy loam and their low contents of organic matter. On the other hand, the old alluvial soil (profile No. 1 \& 2) ranged from 8.8 to $16.1 \mathrm{meq} / 100 \mathrm{~g}$ attributed to their relatively light to medium texture, i.e. loamy sand to sandy loam and very low contents of organic matter. 
THE RELATION BETWEEN THE SOIL TAXONOMIC UNITS ...

TABLE 1. Exchangeable cations, cation exchange capacity and exchangeable sodium

\begin{tabular}{|c|c|c|c|c|c|c|c|c|c|}
\hline \multirow[t]{2}{*}{$\begin{array}{c}\text { Profile } \\
\text { No. }\end{array}$} & \multirow[t]{2}{*}{$\begin{array}{c}\text { Depth } \\
\text { (cm) }\end{array}$} & \multicolumn{4}{|c|}{$\begin{array}{l}\text { Exchangeable cataion } \\
\quad(\mathrm{meq} / \mathbf{1 0 0 g} \text { soil })\end{array}$} & \multirow[t]{2}{*}{$\begin{array}{c}\text { CEC } \\
\text { (me/100g } \\
\text { soil) } \\
\end{array}$} & \multirow[t]{2}{*}{ ESP } & \multirow[t]{2}{*}{$\begin{array}{c}\text { O.M } \\
\%\end{array}$} & \multirow[t]{2}{*}{$\begin{array}{l}\text { CEC } \\
\text { /clay }\end{array}$} \\
\hline & & $\begin{array}{l}\text { Ex. } \\
\mathrm{Na}\end{array}$ & $\begin{array}{l}\text { Ex. } \\
\text { Ca } \\
\end{array}$ & $\begin{array}{l}\text { Ex. } \\
\text { Mg }\end{array}$ & $\begin{array}{c}\text { Ex. } \\
\text { K }\end{array}$ & & & & \\
\hline \multirow[t]{2}{*}{1} & $0-15$ & 5.8 & 5.5 & 3.2 & 1.6 & 16.1 & 36.0 & 1.30 & 0.61 \\
\hline & $15-35$ & 3.1 & 5.1 & 3.5 & 0.6 & 12.3 & 25.0 & 0.15 & 0.70 \\
\hline \multirow{2}{*}{2} & $0-10$ & 1.3 & 5.3 & 1.5 & 0.7 & 8.8 & 15.0 & 1.10 & 0.95 \\
\hline & $10-30$ & 3.1 & 5.1 & 3.1 & 1.1 & 17.4 & 25.0 & 1.40 & 1.10 \\
\hline \multirow{2}{*}{3} & $0-25$ & 1.3 & 0.6 & 0.6 & 0.3 & 2.5 & 0.5 & 0.25 & 0.56 \\
\hline & $25-80$ & 1.0 & 0.6 & 0.6 & 0.3 & 2.3 & 0.5 & 0.21 & 0.53 \\
\hline \multirow{4}{*}{4} & $0-15$ & 0.5 & 11.3 & 6.7 & 0.3 & 28.8 & 13.0 & 2.10 & 0.63 \\
\hline & $15-50$ & 0.8 & 15.3 & 8.1 & 0.5 & 24.7 & 3.0 & 2.00 & 0.73 \\
\hline & $50-80$ & 0.9 & 15.1 & 11.5 & 0.7 & 28.2 & 3.0 & 1.80 & 0.71 \\
\hline & $80-150$ & 1.1 & 15.3 & 7.3 & 0.5 & 24.2 & 4.0 & 1.70 & 0.61 \\
\hline \multirow{4}{*}{5} & $0-20$ & 0.7 & 16.1 & 9.0 & 1.0 & 26.8 & 3.0 & 2.20 & 0.80 \\
\hline & $20-40$ & 0.7 & 14.3 & 9.7 & 1.1 & 25.8 & 3.0 & 2.00 & 0.77 \\
\hline & $40-70$ & 0.8 & 15.1 & 5.3 & 0.7 & 21.9 & 4.0 & 2.00 & 0.77 \\
\hline & $70-150$ & 0.7 & 15.1 & 3.3 & 0.2 & 19.3 & 8.0 & 1.90 & 0.63 \\
\hline \multirow{2}{*}{6} & $0-20$ & 1.3 & 16.0 & 10.3 & 0.8 & 27.4 & 4.0 & 2.35 & 0.70 \\
\hline & $20-40$ & 1.3 & 16.1 & 10.7 & 0.8 & 28.9 & 4.0 & 2.10 & 0.70 \\
\hline \multirow{2}{*}{7} & $0-15$ & 1.5 & 16.5 & 10.3 & 1.5 & 29.8 & 5.0 & 2.30 & 0.73 \\
\hline & $15-40$ & 1.5 & 17.3 & 10.1 & 1.3 & 30.2 & 5.8 & 2.20 & 0.71 \\
\hline \multirow{2}{*}{8} & $0-20$ & 0.4 & 11.4 & 12.1 & 0.2 & 24.1 & 3.0 & 2.25 & 0.67 \\
\hline & $20-40$ & 0.2 & 10.9 & 3.1 & 0.1 & 14.3 & 1.0 & 2.10 & 0.79 \\
\hline \multirow{4}{*}{9} & $0-10$ & 0.3 & 19.1 & 15.3 & 0.5 & 35.2 & 1.0 & 2.30 & 0.60 \\
\hline & $10-55$ & 0.3 & 13.1 & 13.1 & 0.7 & 27.0 & 1.0 & 2.15 & 0.55 \\
\hline & $55-75$ & 0.5 & 8.3 & 15.3 & 0.71 & 24.81 & 2.0 & 2.00 & 0.50 \\
\hline & $75-150$ & 3.0 & 7.0 & 10.1 & 0.5 & 32.6 & 1.0 & 1.70 & 0.66 \\
\hline \multirow{4}{*}{10} & $0-20$ & 3.5 & 15.5 & 13.5 & 1.3 & 35.0 & 10.0 & 2.30 & 0.60 \\
\hline & $20-60$ & 3.3 & 6.1 & 18.5 & 1.1 & 38.0 & 8.0 & 2.20 & 0.67 \\
\hline & $20-110$ & 1.0 & 11.3 & 19.3 & 0.7 & 32.3 & 3.0 & 2.10 & 0.62 \\
\hline & $110-150$ & 3.5 & 11.5 & 19.5 & 0.9 & 35.0 & 10.0 & 1.80 & 0.60 \\
\hline 11 & $0-20$ & 0.5 & 1.5 & 1.0 & 0.3 & 5.40 & 2.0 & 1.90 & 1.03 \\
\hline
\end{tabular}

CEC/ clay

The ratio of CEC/clay of the studied soils ranged from 0.53 to 1.1 (Table 2). The highest ratios were in the old alluvial soils (soil profile No. 2) whereas the lowest were in the windblown soils (profile No. 3). All the studied soils had ratio more than 0.53 indicating the occurrence of the smectitic minerals in the clay particles. The abnormal high ratio in the soil profile No. 2 of the old alluvial reached to 1.1 which may be due to the presence of zeolite minerals which have values of CEC more than $300 \mathrm{meq} / 100 \mathrm{~g}$ (Al-Sharif, 1994). 
TABLE 2. Morphological description of the studied soil profiles.

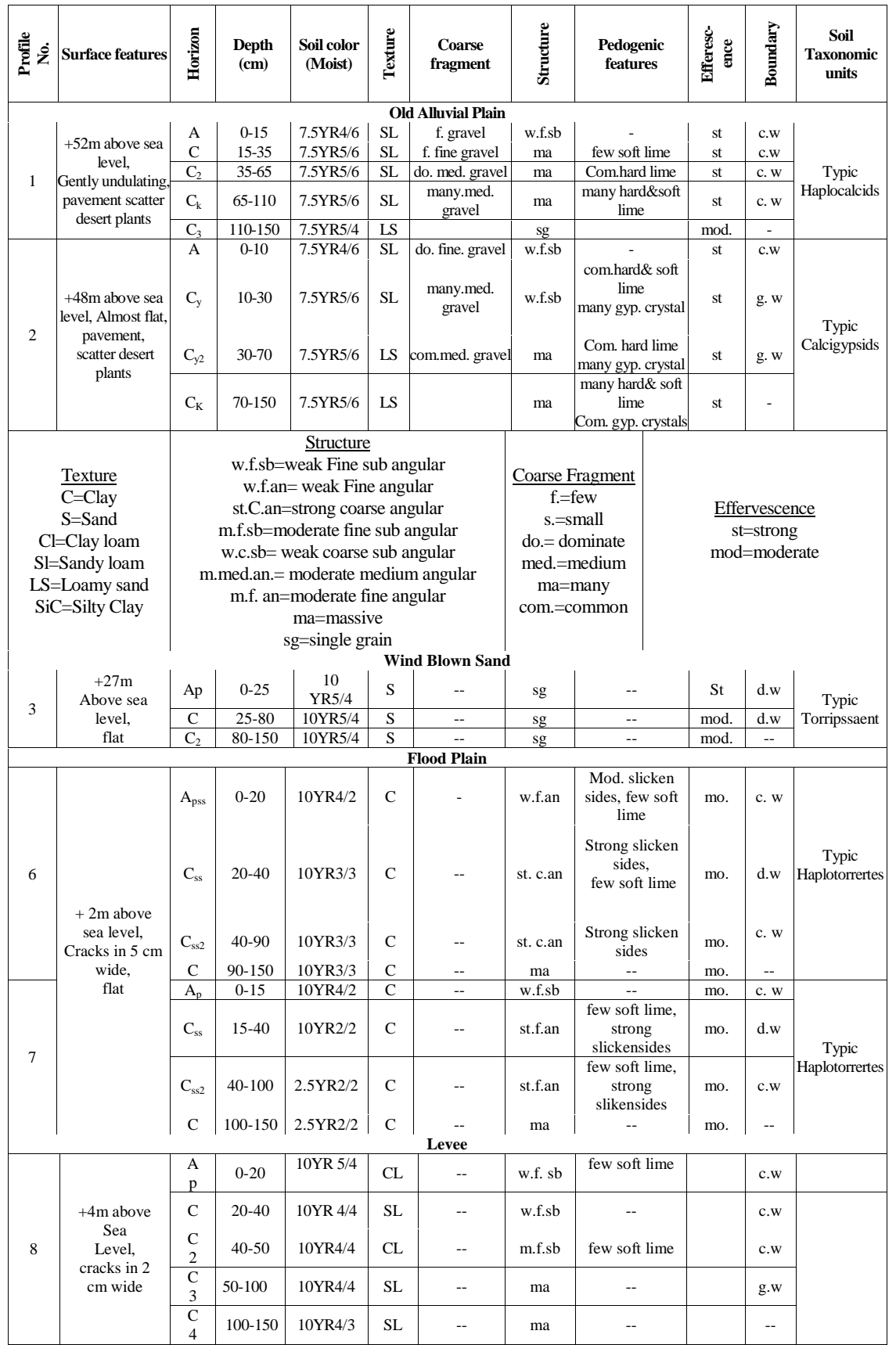

Egypt. J. Soil. Sci. 55, No. 1 (2015) 
TABLE 2. Cont.

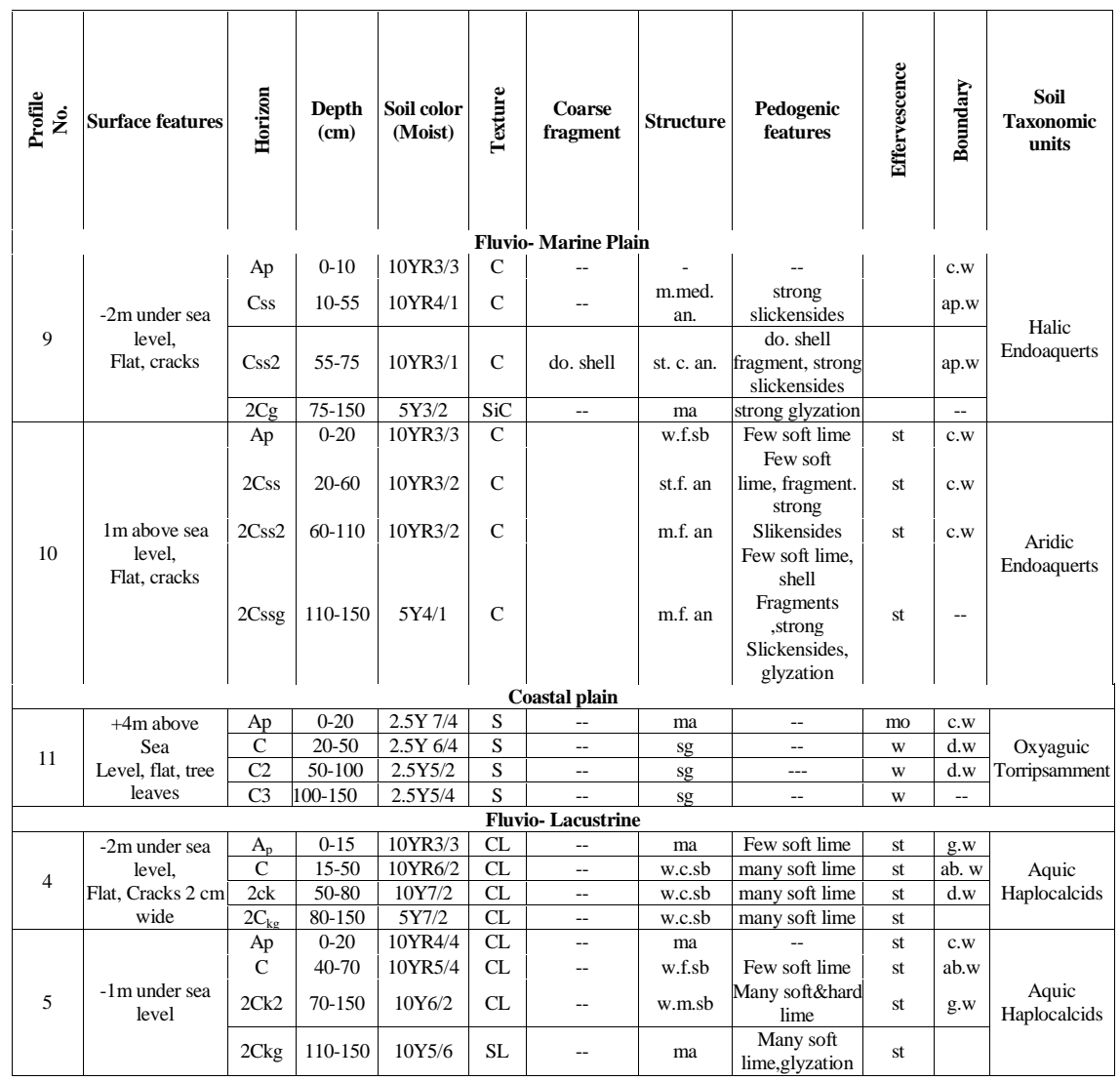

Soil taxonomy of the studied soils

The old alluvial soils

The field description (Table 2) and particle size distribution (Table 3) showed that these soils have sandy loam texture in the soil profile No. 1 in the control section (depth of 20-100 cm), whereas, the clay contents ranged from 18.17 to $20.53 \%$. Also, the soil profile No.2 has loamy sand texture, whereas clay contents ranged from 8.86 to $10.66 \%$ and silt contents reached to $15.43 \%$ both of them has many hard \& soft lime in the $\mathrm{Ck}$ horizon, whereas the content of calcium carbonate reached $16.3 \%$, (Table 3) which qualified as calcic horizon (Keys to Soil Taxonomy, 2010). Therefore, it is classified as fine loamy, mixed, thermic, Typic Haplocalcids. In addition, the soil profile No. 2 has many gypsum crystal, whereas the gypsum contents reached $15.8 \%$ (Table 4) which is qualified as gypsic horizon in the Cy \& Cyz horizon and classified as sandy , mixed, thermic, Typic Calcigypsids (Table 5), and (Map 2). This is in agreement with Al-Sharif et al. (2013). 
TABLE 3. Particle Size distribution, texture class and $\mathrm{CaCO}_{3}$ content of the studied soil profiles.

\begin{tabular}{|c|c|c|c|c|c|c|c|c|}
\hline \multirow{2}{*}{$\begin{array}{l}\text { Prof. } \\
\text { No. }\end{array}$} & \multirow{2}{*}{$\begin{array}{l}\text { Depth } \\
\text { Cm }\end{array}$} & \multirow{2}{*}{$\underset{\%}{\text { Gravel }}$} & \multicolumn{4}{|c|}{ Particle size distribution \% } & \multirow{2}{*}{$\begin{array}{l}\text { Text. } \\
\text { Class }\end{array}$} & \multirow{2}{*}{$\underset{\%}{\mathrm{CaCO}_{3}}$} \\
\hline & & & C.S & F.S & Silt & clay & & \\
\hline \multicolumn{9}{|c|}{ Old Alluvial Plain } \\
\hline \multirow{5}{*}{1} & $0-15$ & - & 18.12 & 27.58 & 27.13 & 27.17 & CL & 5.8 \\
\hline & $15-35$ & - & 42.43 & 21.17 & 18.23 & 18.17 & SL & 5.5 \\
\hline & $35-65$ & - & 32.53 & 31.47 & 15.47 & 20.53 & SL & 10.0 \\
\hline & $65-110$ & - & 42.95 & 26.25 & 12.11 & 18.79 & SL & 15.9 \\
\hline & $110-150$ & - & 67.56 & 13.23 & 12.12 & 7.09 & LS & 4.0 \\
\hline \multirow{4}{*}{2} & $0-10$ & - & 14.75 & $62.4^{\circ}$ & 13.56 & 9.24 & SL & 8.5 \\
\hline & $10-30$ & - & 23.76 & 57.04 & 8.54 & 10.66 & $\mathrm{LS}$ & 6.0 \\
\hline & $30-70$ & - & 42.05 & 33.75 & 15.43 & 8.86 & LS & 6.9 \\
\hline & $70-150$ & - & 36.11 & 43.89 & 10.0 & 10.0 & $\mathrm{LS}$ & 16.3 \\
\hline \multicolumn{9}{|c|}{ Wind Blown Sand } \\
\hline \multirow{3}{*}{3} & $0-25$ & - & 42.26 & 48.94 & 4.4 & 4.4 & $\mathrm{~S}$ & 2.0 \\
\hline & $25-80$ & - & 52.34 & 38.06 & 5.2 & 4.4 & $\mathrm{~S}$ & 1.9 \\
\hline & $80-150$ & - & 45.87 & 44.53 & 4.8 & 4.8 & $\mathrm{~s}$ & 2.0 \\
\hline \multicolumn{9}{|c|}{ Fluvio Lacustrine plain } \\
\hline \multirow{4}{*}{4} & $0-15$ & - & 4.50 & 21.13 & 37.57 & 36.80 & $\mathrm{CL}$ & 12.9 \\
\hline & $15-50$ & - & 1.27 & 34.73 & 30.49 & 33.51 & $\mathrm{CL}$ & 18.0 \\
\hline & $50-80$ & - & 2.05 & 20.35 & 38.36 & 39.24 & $\mathrm{CL}$ & 29.7 \\
\hline & $80-150$ & - & 2.70 & 18.47 & 39.23 & 39.60 & $\mathrm{CL}$ & 26.5 \\
\hline \multirow{4}{*}{5} & $0-20$ & - & 5.22 & 28.55 & 32.77 & 33.46 & $\mathrm{CL}$ & 8.5 \\
\hline & $20-40$ & - & 4.82 & 30.38 & 31.2 & 33.6 & $\mathrm{CL}$ & 10.0 \\
\hline & $40-70$ & - & 6.04 & 36.76 & 28.8 & 28.4 & CL & 19.0 \\
\hline & $70-150$ & & 3.24 & 41.76 & 25.0 & 30.0 & $\mathrm{CL}$ & 23.5 \\
\hline \multicolumn{9}{|c|}{ Flood plain } \\
\hline \multirow{4}{*}{6} & $0-20$ & - & 2.20 & 11.0 & 43.16 & 43.64 & $\mathrm{C}$ & 2.0 \\
\hline & $20-40$ & - & 1.08 & 10.92 & 43.65 & 44.35 & $\mathrm{C}$ & 2.5 \\
\hline & $40-90$ & - & 1.48 & 9.22 & 45.3 & 44.0 & $\mathrm{C}$ & 2.5 \\
\hline & $90-150$ & - & 1.30 & 10.11 & 41.49 & 47.10 & $\mathrm{C}$ & 2.5 \\
\hline \multirow{4}{*}{7} & $0-15$ & - & 1.43 & 12.53 & 40.04 & 46.00 & $\mathrm{C}$ & 2.5 \\
\hline & $15-40$ & - & 1.65 & 12.73 & 30.20 & 55.42 & $\mathrm{C}$ & 3.3 \\
\hline & $40-100$ & - & 2.40 & 10.10 & 30.74 & 56.76 & $\mathrm{C}$ & 2.0 \\
\hline & $100-150$ & - & 2.35 & 13.65 & 24.00 & 60.00 & $\mathrm{C}$ & 7.7 \\
\hline \multicolumn{9}{|c|}{ Fluvio- Marine Plain } \\
\hline \multirow{4}{*}{9} & $0-10$ & - & 0.90 & 6.50 & 34.60 & 58.00 & $\mathrm{C}$ & 2.9 \\
\hline & $10-55$ & - & 0.45 & 7.65 & 32.70 & 59.20 & $\mathrm{C}$ & 3.6 \\
\hline & $55-75$ & - & 0.91 & 5.39 & 32.70 & 61.00 & $\mathrm{C}$ & 4.0 \\
\hline & $75-150$ & - & 0.70 & 3.94 & 75.96 & 19.40 & SiL & 4.0 \\
\hline \multirow{4}{*}{10} & $0-20$ & - & 5.50 & 6.90 & 30.40 & 57.20 & $\mathrm{C}$ & 4.0 \\
\hline & $20-60$ & - & 1.60 & 5.50 & 37.00 & 55.90 & $\mathrm{C}$ & 3.9 \\
\hline & $60-110$ & - & 7.45 & 5.53 & 35.00 & 52.02 & $\mathrm{C}$ & 3.2 \\
\hline & $110-150$ & - & 0.85 & 7.15 & 33.00 & 59.00 & $\mathrm{c}$ & 2.5 \\
\hline \multicolumn{9}{|c|}{ Coastal plain } \\
\hline & $0-20$ & - & 29.25 & 66.25 & 1.30 & 3.20 & $\mathrm{~S}$ & 4.9 \\
\hline & $20-50$ & - & 46.0 & 45.1 & 4.80 & 4.10 & $\mathrm{~S}$ & 5.2 \\
\hline 11 & $50-100$ & - & 39.25 & 51.85 & 3.60 & 5.30 & $\mathrm{~S}$ & 1.3 \\
\hline & $100-150$ & & 35.45 & 57.65 & 1.80 & 5.10 & $S$ & 2.9 \\
\hline
\end{tabular}

C: Clay S: Sandy CL: Clay loam Si: Silty $\quad$ SL: Sandy loam $\quad$ LS: Loamy sand

Egypt. J. Soil. Sci. 55, No. 1 (2015) 
THE RELATION BETWEEN THE SOIL TAXONOMIC UNITS ...

TABLE 4. Soil chemical analysis of the studied soils and gypsum contents.

\begin{tabular}{|c|c|c|c|c|c|c|c|c|c|c|c|c|c|c|c|c|}
\hline \\
\hline \multirow{2}{*}{ 楚 } & \multirow{2}{*}{\multicolumn{2}{|c|}{$\begin{array}{c}\text { Depth } \\
\text { Cm }\end{array}$}} & \multirow{2}{*}{$\begin{array}{l}\text { SP } \\
\% \\
\end{array}$} & \multirow{2}{*}{ PH } & \multirow{2}{*}{$\begin{array}{c}\mathbf{E C} \\
\left(\mathbf{d S m}^{-1}\right)\end{array}$} & \multicolumn{5}{|c|}{ Anions (meq/L) } & \multicolumn{4}{|c|}{ Cations (meq/L) } & \multirow{2}{*}{$\begin{array}{c}\text { Gyp. } \\
\% \\
\end{array}$} & \multirow{2}{*}{ SAR } \\
\hline & & & & & & $\mathrm{CO}_{3}$ & $\mathrm{HCO}_{2}^{-}$ & $\mathrm{Cl}^{-}$ & & $\mathrm{SO}^{=} 4$ & $\mathrm{Ca}^{2+}$ & $\mathrm{Mg}^{2+}$ & $\mathrm{Na}^{+}$ & $\mathbf{K}^{+}$ & & \\
\hline \multirow[t]{7}{*}{1} & $0-15$ & \multicolumn{2}{|l|}{21} & 7.63 & 24.00 & - & 1.69 & 230. & & 38.74 & 74.25 & 22.29 & 160.2 & 1.19 & 0.12 & 35.95 \\
\hline & $15-3$ & 20 & & 7.70 & 31.95 & - & 2.77 & 190. & & 15.60 & 80.61 & 40.74 & 165.83 & 1.19 & 1.58 & 22.04 \\
\hline & $35-6$ & 19 & & 7.84 & 21.91 & - & 1.54 & 240. & & 59.40 & 91.02 & 37.37 & 171.39 & 1.16 & 0.22 & 21.40 \\
\hline & $65-11$ & 110 & & 7.71 & 17.50 & - & 2.00 & 134. & & 61.26 & 76.81 & 19.49 & 99.98 & 0.98 & 1.63 & 14.41 \\
\hline & $110-1$ & 150 & & 7.88 & 16.93 & - & 1.54 & 113. & & 47.47 & 65.35 & 14.86 & 80.94 & 0.86 & 1.57 & 12.79 \\
\hline & $0-10$ & 21 & & 7.43 & 34.50 & - & 1.08 & 200. & & 130.56 & 80.2 & 82.5 & 200.5 & 1.44 & 3.98 & 26.73 \\
\hline & $10-3$ & 30 & & 7.31 & 56.50 & - & 1.38 & 400. & & 200.96 & 100.0 & 26.62 & 450.7 & 1.02 & 15.65 & $90 / 85$ \\
\hline 2 & $30-7$ & 28 & & 7.59 & 79.01 & - & 1.54 & 620. & & 22.40 & 205.13 & 91.16 & 511.1 & 1.28 & 15.81 & 85.95 \\
\hline & $70-15$ & 150 & & 7.58 & 39.45 & - & 1.51 & 595. & & 3.57 & 266.66 & 116.04 & 215.8 & 1.58 & 8.53 & 15.60 \\
\hline & $0-25$ & 18 & & 8.02 & 0.93 & - & 4.30 & 4.00 & & 1.00 & 5.77 & 0.97 & 2.06 & 0.50 & 4.23 & 1.12 \\
\hline 3 & $25-80$ & 18 & & 8.03 & 1.53 & - & 4.30 & 10.0 & & 0.13 & 7.68 & 1.58 & 4.76 & 0.41 & - & 2.21 \\
\hline & $80-15$ & 150 & & 8.24 & 0.48 & - & 3.07 & 1.00 & & 0.73 & 1.96 & 1.08 & 1.40 & 0.36 & - & 1.14 \\
\hline & & & & & & & Fluv & io $\mathrm{L}$ & acustri & & & & & & & \\
\hline & & $0-15$ & 65 & 8.02 & 1.26 & - & 6.15 & & .0 & 5.33 & 7.05 & 5.29 & \begin{tabular}{|l|}
6.98 \\
\end{tabular} & 1.16 & 0.25 & 2.81 \\
\hline & & 15-50 & 70 & 8.12 & 1.61 & - & 3.84 & & 3.0 & 11.09 & 5.3 & 0.84 & 11.15 & 1.05 & 0.14 & 12.46 \\
\hline & 4 & $50-80$ & 60 & 8.06 & 1.46 & - & 3.08 & & .0 & 2.32 & 1.77 & 3.40 & 9.14 & 1.09 & - & 10.58 \\
\hline & & $80-150$ & 60 & 8.02 & 1.45 & - & 3.77 & & .0 & 5.69 & 1.77 & 3.40 & \begin{tabular}{|l|}
9.33 \\
\end{tabular} & 0.96 & 0.18 & 6.48 \\
\hline & & $0-20$ & 55 & 7.87 & 1.51 & - & 4.61 & & 3.0 & 16.10 & 5.03 & 3.10 & \begin{tabular}{|l|}
8.62 \\
\end{tabular} & 0.96 & 0.12 & 4.41 \\
\hline & 5 & $20-40$ & 60 & 7.97 & 1.09 & - & 3.38 & & 00 & 4.25 & 4.33 & 1.16 & \begin{tabular}{|l|}
4.66 \\
\end{tabular} & 0.48 & 0.26 & 2.33 \\
\hline & 5 & $40-70$ & 43 & 7.97 & 1.50 & - & 3.08 & & 00 & 5.39 & 2.48 & 4.63 & \begin{tabular}{|l|}
7.93 \\
\end{tabular} & 0.43 & 0.28 & 4.22 \\
\hline & & $70-150$ & 47 & 8.00 & 1.27 & - & 3.84 & & 00 & 4.72 & 2.84 & 4.03 & 5.35 & 0.34 & 0.17 & 2.89 \\
\hline & & & & & & & Flood & d plai & & & & & & & & \\
\hline & & $0-20$ & 75 & 7.79 & 1.15 & - & 5.84 & 6 & .0 & 12.95 & 10.89 & 0.83 & 12.83 & 0.24 & 0.24 & 5.30 \\
\hline & & $20-40$ & 85 & 8.15 & 1.53 & - & 4.61 & 5 & .0 & 12.48 & 3.84 & 6.03 & 12.19 & 0.03 & 0.13 & 5.49 \\
\hline & 6 & $40-90$ & 72 & 8.12 & 1.51 & - & 3.84 & 5. & 00 & 6.60 & 3.21 & 2.96 & \begin{tabular}{|l|}
8.79 \\
\end{tabular} & 0.48 & 0.17 & 4.99 \\
\hline & & $90-150$ & 65 & 8.01 & 1.45 & - & 4.38 & 5. & 00 & 5.34 & 3.85 & 1.09 & 9.16 & 0.62 & 0.18 & 5.83 \\
\hline & & $0-15$ & 70 & 7.70 & 3.06 & - & 4.15 & 8 & .0 & 18.05 & 10.46 & 5.68 & 13.45 & 0.61 & 0.31 & 4.73 \\
\hline & & 15-40 & 105 & 8.10 & 3.54 & - & 4.15 & 20 & .2 & 11.05 & 4.48 & 7.86 & 22.81 & 0.25 & 0.3 & 9.20 \\
\hline & 7 & $40-100$ & 105 & 8.17 & 3.79 & - & 4.38 & 12 & 2.0 & 18.81 & 4.48 & 6.63 & 23.81 & 0.27 & 0.1 & 10.08 \\
\hline & & 100-150 & 115 & 8.16 & 3.02 & - & 3.99 & 13 & 3.0 & 12.13 & 3.21 & 3.58 & 21.90 & 0.43 & 0.25 & 11.90 \\
\hline & & & & & & & Fluvio- M & Iarine & Plain & & & & & & & \\
\hline & & $0-10$ & & 100 & 7.57 & 2.80 & - & .54 & 15.0 & 7.52 & 12.18 & 1.40 & 15.87 & 0.61 & 0.31 & 6.05 \\
\hline & 9 & $10-55$ & & 110 & 7.90 & 3.70 & - & .34 & 34.0 & 0.25 & 12.18 & 1.40 & 23.17 & 0.84 & 0.08 & 8.88 \\
\hline & 9 & 55-75 & & 115 & 7.89 & 7.69 & - & 61 & 69.0 & 0.92 & 12.82 & 9.40 & 49.20 & 1.11 & 0.021 & 14.80 \\
\hline & & $75-150$ & & 115 & 7.87 & 20.25 & - & 15 & 215.0 & 5.45 & 11.54 & 10.9 & 200.5 & 2.66 & .20 & 84.14 \\
\hline & & $0-20$ & & 100 & 8.00 & 1.67 & - & 15 & 6.0 & 4.32 & 3.21 & 2.34 & 8.99 & 1.93 & 0.16 & 5.42 \\
\hline & 10 & 20-60 & & 120 & 8.05 & 1.85 & - & .38 & 5.0 & 8.28 & 1.92 & 3.63 & 11.74 & 1.37 & 0.26 & 7.07 \\
\hline & 10 & $60-110$ & & 120 & 8.30 & 2.44 & 4. & .15 & 12.0 & 7.06 & 1.92 & 3.02 & 17.45 & 0.82 & 0.15 & 11.11 \\
\hline & & $110-15$ & & 120 & 8.18 & 2.84 & - & 61 & 15.0 & 1.60 & 1.92 & 3.02 & 15.36 & 0.91 & 0.15 & 9.78 \\
\hline & & & & & & & & evee & & & & & & & & \\
\hline & & $0-20$ & & 65 & 7.71 & 1.15 & - & .45 & 4.0 & 3.58 & 8.23 & 0.94 & 2.45 & 0.41 & 0.18 & 1.14 \\
\hline & & $20-40$ & & 60 & 7.60 & 1.53 & - & .38 & 8.0 & 8.22 & 12.8 & 0.78 & 6.50 & 0.52 & 0.28 & 2.49 \\
\hline & 8 & $40-50$ & & 60 & 7.82 & 0.82 & - & .08 & 3.0 & 2.38 & 3.21 & 2.04 & 3.01 & 0.20 & 0.18 & 1.86 \\
\hline & & $50-100$ & & 55 & 7.89 & 0.83 & - & .23 & 3.0 & 1.91 & 3.10 & 0.71 & 4.28 & 0.05 & 0.11 & 3.10 \\
\hline & & $100-15$ & & 60 & 7.91 & 1.69 & - & .15 & 10.0 & 1.45 & 3.21 & 0.60 & 9.68 & 1.11 & 0.15 & 7.01 \\
\hline & & & & & & & Coast: & ta & & & & & & & & \\
\hline & & $0-20$ & & 30 & 7.88 & 1.60 & - & .84 & 7.0 & 15.16 & 8.88 & 3.80 & 5.57 & 0.75 & 0.33 & 2.97 \\
\hline & & $20-50$ & & 30 & 8.02 & 1.13 & - & 61 & 7.0 & 1.61 & 2.56 & 7.32 & 3.09 & 0.25 & 0.19 & 1.39 \\
\hline & 11 & $50-100$ & & 33 & 8.12 & 1.33 & - & .53 & 8.0 & 3.47 & 6.40 & 1.62 & 6.50 & 0.48 & 0.20 & 3.25 \\
\hline & & $100-15$ & & 33 & 8.05 & 1.66 & - & .85 & 10.0 & 2.70 & 3.77 & 2.87 & 9.20 & 0.71 & 0.13 & $\begin{array}{l}5.05 \\
\end{array}$ \\
\hline
\end{tabular}

Egypt. J. Soil. Sci. 55, No. 1 (2015) 


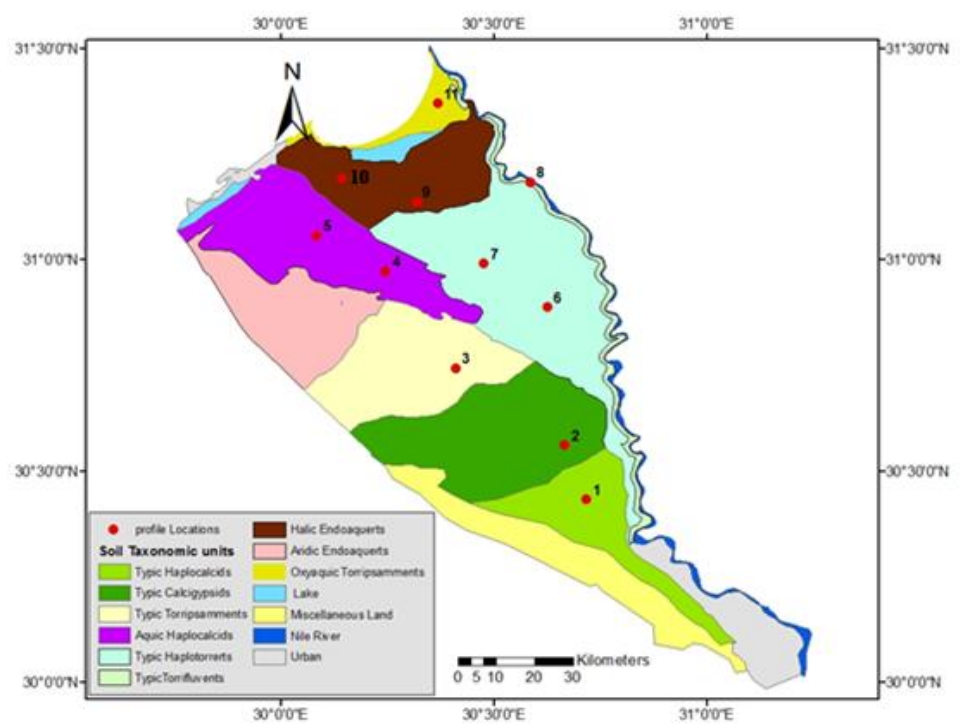

Map 2. Soil taxonomic units of the studied area in Western Delta, Egypt.

TABLE 5. Soil taxonomy of the studied area.

\begin{tabular}{|c|c|c|c|c|c|}
\hline $\begin{array}{l}\text { Pr. } \\
\text { No. }\end{array}$ & Family & Subgreat Group & Great group & Suborder & Order \\
\hline \multicolumn{6}{|c|}{ Old alluvial soils } \\
\hline 1 & $\begin{array}{l}\text { Fine-loamy, mixed, } \\
\text { thermic }\end{array}$ & Typic Haplocalcids & Haplocalcids & Calcids & Aridisol \\
\hline 2 & $\begin{array}{l}\text { Sandy, Mixed, } \\
\text { thermic }\end{array}$ & Typic Calcigypsids & Calcigypsids & Gypsids & Aridisol \\
\hline \multicolumn{6}{|c|}{ Windblown soils } \\
\hline 3 & Siliceous thermic & $\begin{array}{c}\text { Typic } \\
\text { Torripsamment }\end{array}$ & Torripsamment & Psamments & Entiosols \\
\hline \multicolumn{6}{|c|}{ Fluvio lacustrine soils } \\
\hline $4 \& 5$ & $\begin{array}{l}\text { Fine loamy, mixed, } \\
\text { thermic }\end{array}$ & Aquic Haplocalcids & Haplocalcids & Calcidis & Aridisol \\
\hline \multicolumn{6}{|c|}{ Flood plain soils } \\
\hline $6 \& 7$ & $\begin{array}{l}\text { Fine, Smectitic, } \\
\text { thermic }\end{array}$ & Typic Ha[lotorrerts & Haplotorrerts & Torrerts & Vertisol \\
\hline \multicolumn{6}{|c|}{ Levee } \\
\hline 8 & $\begin{array}{c}\text { Coarse loamy, mixed, } \\
\text { thermic }\end{array}$ & Typic Torrifluvents & Torrifluvents & Fluvents & Entisol \\
\hline \multicolumn{6}{|c|}{ Fluvio marine } \\
\hline 9 & $\begin{array}{l}\text { Fine, Smectitic, } \\
\text { thermic }\end{array}$ & Halic Endoaquerts & Edqauerts & Aquerts & Vertisol \\
\hline 10 & $\begin{array}{l}\text { Fine, smectitic, } \\
\text { thermic }\end{array}$ & Aridic Endoaquerts & Edqauerts & Aquerts & Vertisol \\
\hline \multicolumn{6}{|c|}{ Coastal plain } \\
\hline 11 & $\begin{array}{c}\text { Sandy, b Siliceous, } \\
\text { thermic }\end{array}$ & $\begin{array}{c}\text { Oxyaquic } \\
\text { Torripsamments }\end{array}$ & Torripsaments & Psamments & Entisol \\
\hline
\end{tabular}

Egypt. J. Soil. Sci. 55, No. 1 (2015) 


\section{Windblown sand soils}

The studied windblown soil (profile No.3) has sandy texture whereas the sand contents reached 91.0 and no any pedogenic features of carbonates, gypsum or salic, so this soil is classified as siliceous, thermic, Typic Torripasmments.

\section{Fluvio-lacustrine}

Studied soil profiles No. $4 \& 5$ have clay loam texture whereas the clay contents ranged from 23.5 to $30 . \%$ and many soft \& hard lime, calcium carbonate ranged from 18.0 to 29.7 in $2 \mathrm{Ck}$ and $2 \mathrm{Ck} 2$ horizons qualified as Calcic Horizon on elevation of 0 to -2 under sea level, therefore classified as fine loamy, mixed, thermic, Aquic Haplocalcids.

Flood plain of recent Nile alluvial soils

Soil profiles No. $6 \& 7$ had cracks more than $5 \mathrm{~cm}$ width and more than $50 \mathrm{~cm}$ in depth , clay contents ranged from 43.6 to $60.0 \%$ and slickensides in Css and Css2 horizon qualified as fine, smectitic, thermic , Typic Haplotorrerts.

Levee (recent Nile alluvial soils)

Soil profile No. 8 has sandy loam texture alluvial nature and no any pedogenic feature, therefore it is classified as coarse-loamy, mixed, thermic, Typic Torrifluvents.

Fluvio-marine soils

Profiles No $9 \& 10$ have clayey texture , whereas, the clay contents ranged from 52.0 to 61.0 slickensides in the 2 Css \& 2Css 2 horizons, elevations of 0.0 to -2 under sea level. In addition, the soil profile No 9 had ECe of 20.25 in $2 \mathrm{Cg}$ horizon qualified as fine, smectitic, thermic, Halic Endaquerts. Whereas, the studied soil profile No. 10 is fine, smectitic, thermic, Aridic Endaquaerts.

\section{Coastal plain}

Studied soil profile No. 11 has a sandy texture, the sand contents reached $97.0 \%$ and some subsurface horizon are saturated with water, therefore it is classified as sandy, siliceous, thermic, Oxyaquic Torripsamments.

\section{Trace elements contents}

The surface and subsurface horizons of the studied profiles in each soil geomorphic unit were examined to study the normal distribution and variability of several trace elements, i.e., $\mathrm{Fe}, \mathrm{Mn}, \mathrm{Zn}$ and $\mathrm{Cu}$ within and between profiles (Table 6).

The status of iron $(\mathrm{Fe})$

Total Fe contents: It ranged from $0.2 \%$ to $1.2 \%$. The lowest content was in $\mathrm{C} 2$ horizon of the windblown soil whereas, the highest contents were in the Css horizon of the (recent Nile alluvial soils), flood plain and levee soil profile No. 6 and 8, (Table 6). Almost similar iron contents in Egyptian soils were obtained by Hegazy (1980), El-Sayad (1983), El-Toukhy (1987) and Badwi (1999). It is noticeable that the high contents of $\mathrm{Fe}$ in the studied soils are related to both high contents of clay and its semectitic clay mineralogy which contain high contents 
TABLE 6. Total and available contents of some trace elements in the studied soils in $\mathrm{mg} / \mathrm{kg}$.

\begin{tabular}{|c|c|c|c|c|c|c|c|c|c|c|c|c|c|c|}
\hline \multirow[b]{2}{*}{$\begin{array}{c}\text { Profile } \\
\text { No. }\end{array}$} & \multirow[b]{2}{*}{ Horizon } & \multirow[b]{2}{*}{ Depth } & \multicolumn{3}{|c|}{$\mathrm{Fe}$} & \multicolumn{3}{|c|}{ Mn } & \multicolumn{3}{|c|}{$\mathbf{Z n}$} & \multicolumn{3}{|c|}{$\mathrm{Cu}$} \\
\hline & & & *To. \% & *Av. & $\begin{array}{c}\text { Av/To } \\
\%\end{array}$ & To. & Av. & $\begin{array}{c}\mathrm{Av} / \mathrm{To} \\
\%\end{array}$ & To. & Av. & $\begin{array}{c}\text { Av/To } \\
\%\end{array}$ & To. & Av. & $\begin{array}{c}\text { Av/To } \\
\%\end{array}$ \\
\hline \multicolumn{15}{|c|}{ Old Aluvial } \\
\hline \multirow{5}{*}{1} & A & $15-0$ & 0.57 & 4.48 & 0.001 & \begin{tabular}{|l|l|}
316.0 \\
\end{tabular} & 3.12 & 0.007 & 55.6 & 2.24 & 0.05 & 30.0 & 4.50 & 0.2 \\
\hline & $\mathrm{C}$ & $35-15$ & 0.40 & 2.4 & 0.001 & 292.0 & 1.90 & 0.007 & 58.0 & 1.36 & 0.03 & 46.0 & 4.76 & 0.13 \\
\hline & $\mathrm{C}_{2}$ & $65-35$ & 0.56 & 3.04 & 0.001 & 324.0 & 2.78 & 0.01 & 47.4 & 0.78 & 0.02 & 68.0 & 3.96 & 0.07 \\
\hline & $C_{k}$ & $110-65$ & 0.36 & 2.40 & 0.001 & 246.0 & 2.32 & 0.01 & 99.4 & 0.48 & 0.01 & 60.0 & 3.06 & 0.02 \\
\hline & $\mathrm{C}_{3}$ & $150-110$ & 0.25 & 2.40 & 0.001 & \begin{tabular}{|l|}
170.0 \\
\end{tabular} & 1.90 & 0.01 & 83.6 & 0.62 & 0.01 & 72.0 & 4.14 & 0.04 \\
\hline \multirow{4}{*}{2} & $\mathrm{~A}$ & $10-0$ & 0.53 & 3.04 & 0.001 & 216.0 & 2.32 & 0.002 & 85.8 & 0.48 & 0.015 & 42.0 & 4.04 & 0.1 \\
\hline & $\mathrm{C}_{\mathrm{y}}$ & $30-10$ & 0.25 & 4.24 & 0.001 & 154.0 & 1.30 & 0.01 & 132.0 & 0.96 & 0.01 & 60.0 & 4.28 & 0.07 \\
\hline & $\mathrm{C}_{\mathrm{y} 2}$ & $70-30$ & 0.31 & 2.72 & 0.001 & 246.0 & 2.04 & 0.01 & 106.0 & 1.56 & 0.01 & 50.0 & 3.64 & 0.08 \\
\hline & $\mathrm{C}_{\mathrm{K}}$ & $150-70$ & 0.31 & 4.32 & 0.001 & \begin{tabular}{|l|}
262.0 \\
\end{tabular} & 3.12 & 0.01 & 114.8 & 1.42 & 0.02 & 52.0 & 2.52 & 0.07 \\
\hline \multicolumn{15}{|c|}{ Wind Blown sand } \\
\hline \multirow{3}{*}{3} & Ap & $25-0$ & 0.23 & 2.4 & 0.001 & \begin{tabular}{|l|}
330.0 \\
\end{tabular} & 3.06 & 0.01 & 120.0 & 0.61 & 0.01 & 46.0 & 0.98 & 0.03 \\
\hline & $\mathrm{C}$ & $80-25$ & 0.23 & 3.68 & 0.002 & 230.0 & 2.44 & 0.01 & 139.0 & 0.64 & 0.01 & 48.0 & 1.26 & 0.04 \\
\hline & $\mathrm{C} 2$ & $150-80$ & 0.28 & 2.72 & 0.001 & 216.0 & 3.26 & 0.02 & 123.4 & 0.86 & 0.01 & 36.0 & 0.89 & 0.03 \\
\hline \multicolumn{15}{|c|}{ Fluvio-lacustrine } \\
\hline \multirow{4}{*}{4} & Ap & $15-0$ & 0.91 & 10.88 & 0.001 & \begin{tabular}{|l|}
692.0 \\
\end{tabular} & 8.24 & 0.02 & 119.8 & 1.94 & 0.02 & 82.0 & 5.2 & 0.06 \\
\hline & $\mathrm{C}$ & $50-15$ & 0.89 & 8.80 & 0.001 & 738.0 & 6.46 & 0.03 & 130.6 & 1.24 & 0.02 & 104.0 & 5.76 & 0.05 \\
\hline & ck2 & $80-50$ & 0.80 & 6.08 & 0.001 & \begin{tabular}{|l|}
508.0 \\
\end{tabular} & 5.84 & 0.01 & 177.6 & 0.96 & 0.015 & 48.0 & 4.48 & 0.1 \\
\hline & Ckg2 & $150-80$ & 0.84 & 7.68 & 0.001 & 600.0 & 5.38 & 0.01 & 155.0 & 0.48 & 0.05 & 52.0 & 4.84 & 0.1 \\
\hline \multirow{4}{*}{5} & Ap & $20-0$ & 0.95 & 14.56 & 0.002 & 894.0 & 6.06 & 0.008 & 166.0 & 0.74 & 0.05 & 42.0 & 3.50 & 0.1 \\
\hline & $\mathrm{C}$ & $70-40$ & 0.70 & 15.36 & 0.001 & 800.0 & 6.4 & 0.01 & 162.2 & 0.78 & 0.006 & 22.0 & 2.96 & 0.15 \\
\hline & Ck22 & $150-70$ & 0.65 & 9.76 & 0.001 & 546.0 & 7.48 & 0.02 & 212.6 & 0.48 & 0.005 & 28.0 & 2.88 & 0.15 \\
\hline & Ckg2 & $150-110$ & 0.77 & 8.64 & 0.001 & 524.0 & 10.2 & 0.02 & 213.6 & 0.70 & 0.003 & 50.0 & 2.78 & 0.6 \\
\hline & & & & & & Floo & d & & & & & & & \\
\hline & Apss & $20-0$ & 1.15 & 43.2 & 0.005 & \begin{tabular}{|l|}
632.0 \\
\end{tabular} & 9.52 & 0.02 & 248.8 & 1.28 & 0.01 & 36.0 & 3.14 & 0.1 \\
\hline 6 & Css & $40-20$ & 1.20 & 21.92 & 0.003 & 708.0 & 8.78 & 0.01 & 236.6 & 0.66 & 0.005 & 46.0 & 4.32 & 0.1 \\
\hline 0 & Css2 & $90-40$ & 1.21 & 25.2 & 0.002 & 692.0 & 10.88 & 0.08 & 232.0 & 0.96 & 0.005 & 22.0 & 3.32 & 0.15 \\
\hline & C & $150-90$ & 1.19 & 24.88 & 0.002 & 862.0 & 12.92 & 0.02 & 265.0 & 1.14 & 0.008 & 22.0 & 3.14 & 0.14 \\
\hline & Ap & $15-0$ & 1.17 & 57.6 & 0.005 & 970.0 & 9.32 & 0.01 & 313.4 & 1.94 & 0.003 & 23.0 & 3.68 & 0.14 \\
\hline 7 & Css & $40-15$ & 1.16 & 30.8 & 0.003 & \begin{tabular}{|l|l}
954.0 \\
\end{tabular} & 8.9 & 0.01 & 297.6 & 0.92 & 0.003 & 28.0 & 4.32 & 0.14 \\
\hline 1 & Css2 & $100-40$ & 1.18 & 23.12 & 0.002 & \begin{tabular}{|l|}
908.0 \\
\end{tabular} & 5.98 & 0.01 & 253.4 & 0.74 & 0.004 & 56.0 & 3.68 & 0.06 \\
\hline & $\mathrm{C}$ & \begin{tabular}{|c|}
$150-100$ \\
\end{tabular} & 1.17 & 23.6 & 0.002 & \begin{tabular}{|l|}
786.0 \\
\end{tabular} & 5.30 & 0.01 & 264.0 & 0.92 & 0.004 & 42.0 & 4.14 & 0.1 \\
\hline & & & & & & & evee & & & & & & & \\
\hline & Ap & $20-0$ & 1.20 & 36.8 & 0.004 & \begin{tabular}{|l|l|}
676.0 \\
\end{tabular} & 5.50 & 0.01 & 262.0 & 1.48 & 0.01 & 36.0 & 4.22 & 0.1 \\
\hline & C & $40-20$ & 0.12 & 33.4 & 0.003 & 278.0 & 5.98 & 0.02 & 274.0 & 1.04 & 0.004 & 42.0 & 5.3 & 0.1 \\
\hline 8 & $\mathrm{C} 2$ & $50-40$ & 1.21 & 32.0 & 0.003 & 278.0 & 4.62 & 0.02 & 255.4 & 1.14 & 0.004 & 62.0 & 4.22 & 0.07 \\
\hline & C3 & $100-50$ & 1.14 & 25.6 & 0.002 & 200.0 & 5.84 & 0.03 & 279.8 & 0.92 & 0.003 & 68.0 & 5.22 & 0.08 \\
\hline & $\mathrm{C} 4$ & \begin{tabular}{|c|}
$150-100$ \\
\end{tabular} & 0.97 & 25.6 & 0.003 & \begin{tabular}{|l|}
354.0 \\
\end{tabular} & 6.46 & 0.02 & 308.2 & 1.98 & 0.01 & 104.0 & 5.74 & 0.06 \\
\hline & & & & & & Fluvi & -Marin & & & & & & & \\
\hline & Ap & $10-0$ & 0.85 & 20.4 & 0.003 & 538.0 & 4.9 & 0.01 & 312.6 & 1.78 & 0.01 & 110.0 & 7.56 & 0.07 \\
\hline 0 & Css & $55-10$ & 1.05 & 20.6 & 0.003 & 646.0 & 5.92 & 0.01 & 311.0 & 2.34 & 0.01 & 126.0 & 7.28 & 0.06 \\
\hline 9 & Css2 & $75-55$ & 0.87 & 14.0 & 0.002 & 908.0 & 4.7 & 0.01 & 320.6 & 1.72 & 0.01 & 140.0 & 6.84 & 0.05 \\
\hline & $\mathrm{Cg} 2$ & $150-75$ & 0.59 & 10.8 & 0.003 & 1048.0 & 5.72 & 0.01 & 325.4 & 3.68 & 0.01 & 156.0 & 6.10 & 0.05 \\
\hline & Ap & $20-0$ & 0.41 & 22.4 & 0.006 & 554.0 & 4.96 & 0.01 & 553.2 & 3.26 & 0.01 & 140.0 & 8.36 & 0.06 \\
\hline & Css2 & $60-20$ & 0.46 & 11.68 & 0.004 & \begin{tabular}{|l|}
654.0 \\
\end{tabular} & 4.9 & 0.01 & 327.4 & 2.92 & 0.01 & 156.0 & 8.0 & 0.05 \\
\hline 10 & Css22 & $110-60$ & 0.47 & 6.24 & 0.003 & \begin{tabular}{|l|}
1016.0 \\
\end{tabular} & 5.72 & 0.01 & 350.4 & 2.74 & 0.01 & 168.0 & 9.90 & 0.06 \\
\hline & Cssg2 & \begin{tabular}{|c|}
$150-110$ \\
\end{tabular} & 0.29 & 6.88 & 0.003 & \begin{tabular}{|l|}
862.0 \\
\end{tabular} & 4.96 & 0.01 & 370.4 & 5.22 & 0.01 & 113.0 & 8.04 & 0.1 \\
\hline & & & & & & Coas & tal plaiı & & & & & & & \\
\hline & Ap & $20-0$ & 0.39 & 8.64 & 0.003 & \begin{tabular}{|l|}
570.0 \\
\end{tabular} & 6.26 & 0.01 & 358.6 & 0.92 & 0.01 & 83.0 & 3.72 & 0.05 \\
\hline & $\mathrm{C}$ & $50-20$ & 0.52 & 8.84 & 0.002 & \begin{tabular}{|l|}
678.0 \\
\end{tabular} & 7.48 & 0.01 & 305.2 & 0.62 & 0.01 & 70.0 & 4.04 & 0.05 \\
\hline 11 & $\mathrm{C} 2$ & $100-50$ & 0.46 & 11.52 & 0.003 & \begin{tabular}{|l|}
492.0 \\
\end{tabular} & 6.46 & 0.015 & 341.2 & 1.90 & 0.02 & 74.0 & 3.78 & 0.05 \\
\hline & C3 & \begin{tabular}{|l|}
$150-100$ \\
\end{tabular} & 0.45 & 5.28 & 0.002 & \begin{tabular}{|l|}
524.0 \\
\end{tabular} & 6.4 & 0.01 & 325.0 & 1.02 & 0.01 & 54.0 & 4.02 & 0.08 \\
\hline
\end{tabular}

* To.= Total, Av.= Available

Egypt. J. Soil. Sci. 55, No. 1 (2015) 
of exchangeable $\mathrm{Fe}$ ions between the layers of their allumonosilicates (ElDemerdashe, 1970). Whereas, both Fluvio lacustrine and Fluvio-marine soils, soil profiles No. 4 \& 5, 9 and 10 contained moderate contents ranged from 0.29 to 0.90 related to their nature calcareous material; similar data were obtained by El-Tokhy (1987). Coastal plain, (soil profile No. 11) ranged from $0.39 \%$ to $0.46 \%$, which may be due to the presence of feldspars, (El-Demerdashe, 1970). Old alluvial soils profiles No. $1 \& 2$, and the windblown soils, soil profile No. 3 contained the lowest contents ranged from 0.2 to $0.57 \%$ due to their nature sandy deposits (El-Demerdashe, 1970 and El-Tokhy, (1987).

The partial; equation of total Fe with independent factors of both clay, silt and $\mathrm{CaCO}_{3}$ contents

Typic Haplocalcids and Typic Calcigypsid

Partial regression analysis was performed in order to reveal the relative distribution of total $\mathrm{Fe}$ between clay, silt and $\mathrm{CaCO}_{3}$. The regression equation reads:

Total $\mathrm{Fe}=88.1+23.8($ silt $\%)+54.3($ Clay $\%)+50.8\left(\mathrm{CaCO}_{3}\right)$

This means that $55.6 \%(\mathrm{R} 2=0.556)$ of the variations in total Fe content of the soils could be accounted for the clay, silt and $\mathrm{CaCO}_{3}$ content of the soil (Table 7). The regression coefficient equation meaned that an increase of one percent of either clay or silt, $\mathrm{CaCO}_{3}$ will increase total Fe by 54.3, $23.8 \mathrm{ppm}$ and $50.8 \mathrm{ppm}$, respectively.

\section{Aquic Haplocalcids}

Correlation between total $\mathrm{Fe}$ and clay or silt or $\mathrm{CaCO}_{3} \%$ contents, showed the equation of partial regression for these soils as:

Total $\mathrm{Fe}=3260.0+267\left(\right.$ Clay \%) $-94.65\left(\right.$ silt \%) $-63.95\left(\mathrm{CaCO}_{3}\right)$

This means that $39.1 \%$ of the variations of total $\mathrm{Fe}$ contents could be accounted for clay, silt and $\mathrm{CaCO}_{3} \%$ content of these soils.

\section{Typic Haplotorrerts}

Total $\mathrm{Fe}=1602.3+152.55($ clay $\%)+149.5($ silt $\%)+78.1\left(\mathrm{CaCO}_{3}\right)$

whereas the clay, silt and $\mathrm{CaCO}_{3} \%$ contribute 83.2 of the factors affecting the variation of total Fe.

\section{Typic Torrifluvents}

Total $\mathrm{Fe}=2178.7-1566.2+1942.2($ silt $\%)-626.3\left(\mathrm{CaCO}_{3}\right)$.

whereas, both clay, silt and $\mathrm{CaCO}_{3} \%$ contribute $89.7 \%$ of the factors affecting the variation of total Fe.

Halic Endoaquerts and Aridic Endoaquerts

Total $\mathrm{Fe}=-43573.5+531.8($ clay $\%)+451.4+1196.8($ Clay $\%)$.

The clay, slit and $\mathrm{CaCO}_{3} \%$ contents contribute $28.7 \%$ of the factors affecting the variations of total $\mathrm{Fe}$ in these studied soils. 
TABLE 7. \% of the variation (R2) of the studied total trace elements related to clay, silt and $\mathrm{CaCO}_{3} \%$ in the studied soils.

\begin{tabular}{|c|c|c|c|c|c|c|}
\hline 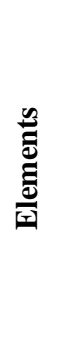 & 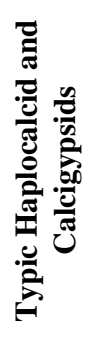 & 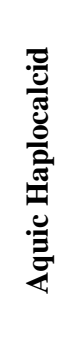 & 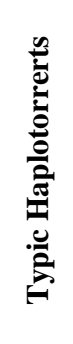 & 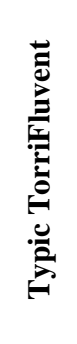 & 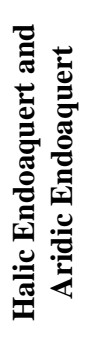 & 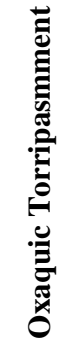 \\
\hline $\mathrm{Fe}$ & 55.6 & 39.1 & 83.1 & 89.7 & 24.8 & 83.0 \\
\hline $\mathrm{Mn}$ & 93.4 & 55.8 & 45.7 & 57.3 & 26.5 & 85.0 \\
\hline $\mathrm{Zn}$ & 58.4 & 67.5 & 51.5 & 88.9 & 49.3 & 81.0 \\
\hline $\mathrm{Cu}$ & 58.7 & 10.9 & 25.3 & 73.3 & 61.7 & 90.0 \\
\hline $\mathrm{Cd}$ & 42.4 & 66.5 & 21.0 & 85.0 & 20.3 & 81.0 \\
\hline
\end{tabular}

Oxyaquic Torripsamments

Total $=945.7+481.9($ clay $\%)+226.0($ silt $\%)+223.3(\mathrm{CaCO} 3)$.

Whereas, both clay, silt and $\mathrm{CaCO}_{3}$ associated with $83.0 \%$ of the factors affecting the variations of total $\mathrm{Fe}$.

Available Fe Contents: It ranged from 2.4 to $57.6 \mathrm{ppm}$. The highest values were in the Typic Haplotorrerts and Typic Torrifluvents, studied soil profile No. 6, 7 and 8 reached $57.6 \mathrm{ppm}$ in Ap horizon of soil profile No. 7. This high content is related to the nature of these alluvial soils rich in exchangeable iron in the allumoniosilicate of the clay minerals in addition to its high CEC resulted from their heavy Aquic Haplocalcids, Halic Endoaquerts and Aridic Endoaquerts clay texture and addition of organic fertilizers. These soils followed by both then the Oxyaquic Torripsamments soils which had nearly contents of the available Fe ranged from 5.28 in C3 horizon of the studied soil profile No. 11 to $20.6 \mathrm{ppm}$ in Css horizon of the soil profile No. 9. Similar values are reported by El-Sayad and El-Tokhy (1987) in these soils. Finley, both the Typic Haplocalcid and Calcigypsids, studied soil profile No. 1, 2 and Typic Torripsamments, soil profile No.3 had the lowest contents of the available Fe ranged from 2.4 to 2.48, related their sandy texture. The percent of available Fe/total Fe from relatively low ranged from 0.001 to $0.006 \%$. The high values were in the Typic Haplotorrerts and Endoaquerts.

The status of Manganese (Mn)

Total manganese contents: It ranged from 170.0 to $1048.0 \mathrm{ppm}$. The highest contents were in the subsurface horizon of the Typic Haplotorrerts, Aquic Haplocalcids, Aridic Endoaquerts and Halic Endoaquerts soil profile No. 4, 5,6, 7, 9 and 10 ranged from 508.0 to 1048.0 ppm (Table 6); similar contents were

Egypt. J. Soil. Sci. 55, No. 1 (2015) 
reported by Taha (1980) and Badwi (1999). This is due to annual deposition of the Nile suspended matter which contains $0.23 \%$ total Mn expressed manganese dioxide $\mathrm{MnO}_{2}$ (Ball, 1952).

The coastal plain studied soil profile No. 11 contained a moderate contents of the total $\mathrm{Mn}$ ranged from to 492.0 to 678.0 related to their nature coastal material. On the other hand, both the old alluvial, (soil profile No. $1 \& 2$ ) and windblown sand soils, (soil profile No. 3) contained low contents of the total Mn ranged from 154.0 to $330.0 \mathrm{ppm}$. The wide variation of total $\mathrm{Mn}$ in the studied soils is due to the variation in their clayey texture as well as entry of Mn into the crystal lattice of the allumonosilicate layers of the clay and the presence of $\mathrm{Mn}$ nodules and concretions and the metal organic complexes (El-Demerdashe, 1970 and Taha, 1980).

The partial; equation of total Mn with the independent factors of both clay, silt and $\mathrm{CaCO}_{3}$ contents:

Typic Haplocalcid and Calcigypsid

Total Mn had a significant correlation with clay $\%$ and $\mathrm{CaCO}_{3}$ in Typic Haplocalcid and Calcigypsid soils, whereas a highly significant correlation with silt. The partial regression equation was:

Total $\mathrm{Mn}=-14.19+2.32($ clay $\%)+12.34($ silt $)+6.51\left(\mathrm{CaCO}_{3}\right)$.

This means that $93.4 \%$ of the factors affecting the variation in total $\mathrm{Mn}$ in these studied soils, related to both contents of the independent factors of clay, silt and $\mathrm{CaCO}_{3}$.

\section{Aquic Haplocalcids}

The partial regression equation was:

Total $\mathrm{Mn}=552.3+37.3\left(\right.$ clay\%) $-27.3($ silt $\%)-15.8 \mathrm{CaCO}_{3}$.

The clay, silt and $\mathrm{CaCO}_{3} \%$ contents of the soils were responsible for $22.7 \%$ of the factors affecting the variations of total $\mathrm{Mn}$.

\section{Typic Haplotorrerts}

Total $\mathrm{Mn}=2784.9-14.9$ (clay \%) -28.7 ( silt) $-50.7\left(\mathrm{CaCO}_{3} \%\right)$.

Whereas, the independent factors of clay, silt $\mathrm{CaCo} 3$ contribute the $45.8 \%$ of the factors affecting the variations of total $\mathrm{Mn}$.

\section{Typic Torrifluvents}

The partial regression was:

Total Mn = $1594.0+85.4\left(\right.$ clay \%) -73.8 (silt)-298.5 $\left(\mathrm{CaCO}_{3}\right)$.

These independent components contributed 57.3 of the factors affecting total $\mathrm{Mn}$ in these soils.

\section{Endoaquerts}


Total $\mathrm{Mn}=782.4-2.62($ clay $\%)+4.7($ silt $)-3.3\left(\mathrm{CaCO}_{3}\right)$.

The studied independent factors contribute $28.8 \%$ of the factors affecting the variation of total $\mathrm{Mn}$ in this studied soils.

Oxyaquic Torripsamments

Total $\mathrm{Mn}=249.0+19.5($ clay\% $)+21.81(\mathrm{Silt} \%)+46.9\left(\mathrm{CaCO}_{3}\right)$.

About 85.0 were related to the independent factors clay. silt and $\mathrm{CaCO}_{3}$ associated the variations of total $\mathrm{Mn}$ in soils.

Available Mn contents: It ranged from 1.3 to $12.92 \mathrm{ppm}$. The Typic Haplotorrerts soils studied soils No. 6, 7 had the highest levels reached 12.92, this may be related to their nature alluvial materials. On the other hand studied soils of Aquic Haplocalcids, Halic Endoaquerts, Aridic Endoaquerts and Oxyaquic Torripsamments had nearly levels whereas the studied soils of Typic Haplocalcids and Typic Calcigypsids (soil profiles No. 1, 2) and Typic Torripsamments, (soil profile No. 3) had the lowest levels ranged from 1.3 to $3.26 \mathrm{ppm}$. The percent of available $\mathrm{Mn} /$ total $\mathrm{Mn}$ ranged from 0.007 to $0.02 \%$. The highest values were in Endoaquerts, due to occurrence of some marine constitutes which contain Mn.

The status of zinc $(\mathrm{Zn})$

Total zinc contents

In the studied area total $\mathrm{Zn}$ ranged from 47.4 to $370.0 \mathrm{ppm}$. These values were closer to that obtained by El-Dermerdashe (1970), Abd El-Wahid (1976) and Badwi (1999).

Aquic Haplocalcids (soil profile No. $9 \& 10$ ) and Oxyaquic Torripsamments (soil profile No. 11) had the highest contents of total $\mathrm{Zn}$ ranged from $305.2 \mathrm{ppm}$ in the soil profile No. 11 to $370.6 \mathrm{ppm}$ in the studied soil profile No. 10 . This is due to their marine nature which contained some constituents rich in $\mathrm{Zn}$, high content of clay and some Feldspar minerals (El-Demerdashe, 1970). The Aquic Haplocalcids soils (soil profile No. 4\&5) had a moderate contents of total $\mathrm{Zn}$ ranged from 119.8 in Ap horizon of studied soil No.4 to 213.6 in the Ck horizon of studied soil profile No. 5, this is related to the calcareous nature of the lacustrine contained $\mathrm{Zn}$ constituents' lower than both marine and recent alluvial deposits and their moderate content of clay, i.e. clay loam texture (Abd El-Wahid, 1976). The presence of $\mathrm{Zn}$ in the windblown (soil No. 3) is due to the added organic matter fertilizers. On the other hand, the Typic Haplocalcids and Typic Calcigypsids (soil profile No.1\& 2) had the lowest values of total $\mathrm{Zn}$ reached 47.4 related to their nature parent materials poor in $\mathrm{Zn}$ and their light texture.

Partial regression equations for total $\mathrm{Zn}$ with independent variable of clay, silt and $\mathrm{CaCO} 3 \%$ contents in the studied soils as:

Typic Haplocalcids and Typic Calcigypsids

Total $\mathrm{Zn}=131.5-1.59$ (clay \%)- $2.05\left(\right.$ silt $\% 0+1.0\left(\mathrm{CaCO}_{3} \%\right)$

Egypt. J. Soil. Sci. 55, No. 1 (2015) 
Both clay, silt and $\mathrm{CaCO} 3 \%$ contents in soils contribute $58.5 \%$ of the factors affecting the variation of total $\mathrm{Mn}$.

Aquic Haplocalcids

Total $\mathrm{Zn}=360.0-8.7\left(\right.$ clay \%) $+1.6($ silt \% $)+2.7\left(\mathrm{CaCO}_{3}\right)$.

The studied independent variables contribute $67.0 \%$ of the factors affecting the variations of total $\mathrm{Zn}$ in these studied soils.

\section{Typic Haplotorrerts}

The partial regression was:

Total $\mathrm{Zn}=1790.4-17.6($ Clay\% $)-17.2($ silt $\%)-10.0\left(\mathrm{CaCO}_{3}\right)$.

Whereas, $52.4 \%$ of the factors affected the variation of total $\mathrm{Zn}$ in this studied soils were related to both clay, silt and $\mathrm{CaCO} 3 \%$ content in soil.

\section{Typic Torrifluvents}

Total $\mathrm{Zn}=436.7+1.6$ (clay\%)-2.9(silt\%)-25.0 $\left(\mathrm{CaCO}_{3} \%\right)$.

Whereas, these independent factors contributed 88.9 of the factors affected the variation of total $\mathrm{Zn}$ in the soils.

Halic Endoaquerts and Aridic Endoaquerts

Total $\mathrm{Zn}=2041.9-20.03$ (clay\%)-19.77 (silt \%)-40.7 $\left(\mathrm{CaCO}_{3}\right)$.

Whereas, the clay, silt and $\mathrm{CaCO} 3 \%$ contents in soils contribute $49.3 \%$ of the factors affected the variation of total $\mathrm{Zn}$ in the studied soil.

\section{Oxyaquic Torripsamments}

Total $\mathrm{Zn}=574-36.5($ clay\% $)-4.0($ silt $\%)-19.2\left(\mathrm{CaCO}_{3} \%\right)$.

The studied independent factors contribute $81 \%$ or all the factors affected the variations of total $\mathrm{Zn}$ in this studied soils.

Available Zn contents: It ranged from 0.48 to $5.22 \mathrm{ppm}$ in the studied soil profiles. The Endoaquerts soils No. 9, 10 had the higher contents ranged from 1.72 to $5.22 \mathrm{ppm}$ due to their marine nature material contained some of $\mathrm{Zn}$ constituents. On the other hand all other studied soils had low levels of the available $\mathrm{Zn}$. The percent of available $\mathrm{Zn} /$ total $\mathrm{Zn}$ ranged from 0.003 to $0.05 \%$.

The status of copper

Total copper contents: It ranged from 22.0 to $168.0 \mathrm{ppm}$. Almost similar values were reported in some Egyptian soils by El-Demerdashe (1970), ElTokhy (1987) and Badwi (1999). The highest contents were in the Endoaquerts soils No. $9 \& 10$ and surface soil (Ap) horizon of both Aquic Haplocalcids and Oxyaquic Torripsamments due to their nature marine deposits rich in $\mathrm{Cu}$ constituents and additional organic fertilizer. On the other hand, the other studied soils had a moderate content. These values are almost similar to which reported by El-Demerdashe (1970), Abd El-Wahid (1976) and El-Toukhy (1987). 
The partial equation of total $\mathrm{Cu}$ with the independent factors of clay, silt and CaCO3 contents

Typic Haplocalcids and Calcigypsids

Total $\mathrm{Cu}=91.7+1.13($ clay\% $)-3.03($ silt $\%)-1.13\left(\mathrm{CaCO}_{3} \%\right)$

Therefore, 58.7 of the variations of total $\mathrm{Cu}$ in these soils could have accounted by clay, silt and $\mathrm{CaCO}_{3}$ in the soils.

Aquic Calcigypsids

Total $\mathrm{Cu}--30.3+5.7$ (clay\%)-3.3 (silt\%)-3.1 $\left(\mathrm{CaCO}_{3} \%\right)$

So; $10.9 \%$ of the variation of total $\mathrm{Cu}$ in this studied soils was related to clay, silt and $\mathrm{CaCO}_{3}$ in the soils.

Typic Haplotorrerts

Total $\mathrm{Cu}=-64.27+1.7$ (clay $\%)-0.47($ silt $\%)-1.7\left(\mathrm{CaCO}_{3} \%\right)$

Whereas, $25.3 \%$ of the variations of total $\mathrm{Cu}$ in this studied soils were related to clay, silt and $\mathrm{CaCO}_{3} \%$.

Typic Torrifluvents

Total $\mathrm{Cu}=205.3-2.7($ clay $\%)+2.1($ silt $\%)-25.9\left(\mathrm{CaCo}_{3} \%\right)$

Whereas, $73.3 \%$ of the variations of total $\mathrm{Cu}$ were related to clay, silt and $\mathrm{CaCO}_{3} \%$ contents in this studied soils.

\section{Endoaquerts}

Total $\mathrm{Cu}=438.1-4.3($ clay\% $)-3.5($ silt $\%)+18.2\left(\mathrm{CaCO}_{3} \%\right)$

Whereas, $61.8 \%$ of the variations of total $\mathrm{Cu}$ in this soils were be accounted to clay, silt and $\mathrm{CaCO}_{3} \%$.

Oxyaquic Torripsamments

Total $\mathrm{Cu}=220.9-28.0\left(\right.$ clay \%) $+4.3($ silt $\%)-11.0\left(\mathrm{CaCO}_{3} \%\right)$

Whereas, 90.0 of the variation of total $\mathrm{Cu}$ in this studied soils were accounted to clay, silt and $\mathrm{CaCO}_{3} \%$ contents.

Available Cu contents: It ranged from 0.89 to $9.9 \mathrm{ppm}$. The studied Aridic, Halic Endoaquerts No. 9, 10 contained the highest levels ranged from 6.1 to 9.9 releated to their marine nature which had some constituents rich in $\mathrm{Cu}$. Whereas, the other soils had a moderate levels ranged from 2.78 to $5.74 \mathrm{ppm}$.

Finally, the Typic Torripsamments soils No. 3 had the lowest levels reached 0.89 to $1.26 \mathrm{ppm}$ releated to their sandy nature.

The obtained data of the total trace elements, for the studied area showed that their vertical distribution does not help in providing specific trends rating to their nature material or geomorphic unit. The differences between the levels of the top and upper subsoil are mostly irregular and thus could be ascribed to any of the local environmental which cause the occurrence of lithological discontinuity between their parent materials which agree with El-Demerdash (1970). 


\section{Soil fertility releated to soil taxonomic units}

The above discussion of both total and available trace elements, i.e. Fe, Mn, $\mathrm{Zn}$ and $\mathrm{Cu}$ revealed that soil Taxonomic Unit of both clayey, smectitic, thermic Typic Haplotorrerts studied soil No 6,7 and the taxonomic unit of clayey, smectitic, thermic. Halic Endaquerts and Aridic Endaquerts, studied soil profile No. $9 \mathrm{~m} 10$ had relatively high contents of these elements. This could be a reflection of their nature of the alluvial and Fluvial-marine material, the heavy texture contained a high clay content rich in smectitic clay mineral which possesses a high cation exchange capacity use of organic manure commercial fertilizers, microbiological enrichment and the moisture regime. Therefore this taxonomic unit is the richest in fertility.

The taxonomic unit of Typic Torrifluvents studied soil No. 8 had contents close to the Typic Haplotorrerts due to the above mentioned reasons.

On the other hand, both Taxonomic units of Fine loamy, mixed thermic Aquic Haplocalcids of the studied soil No. 4, 5 had moderate contents of the studied trace elements related to their nature Fluvio-lacustrine affected by the calcareous characterization and medium texture lower in CEC than the above soils adsorbed lowers contents of plant nutrient.

The studied soil Oxyaquic Torripsaments had also relatively moderate contents of some studied trace elements may be due to the occurrence of some feldspar minerals.

Finally, Taxonomic unit of both sandy, siliceous, thermic, Typic Haplocalcids, and Typic Calcigypisds studied soil profile No. 1, 2 of the old alluvial and sandy, siliceous, thermic, Typic Torripasamments, studied soil profile No. 3 had the lowest contents of the studied trace elements due to their sandy texture as the quartz predominates the other constituents whereas the trace elements contents of quarts are low. Therefore this taxonomic unit is poor in fertility.

According to the previous discussion for the obtained results of this investigation the studied soil taxonomic units can be ordered according to their soil fertility as follows:

Fine, smectitic, thermic, Typic Haplotorrerts (Flood plain) and Fine smectitic thermic, Halic Endoquerts (Fluvio-marine) $>$ Fine loamy, mixed, thermic, Typic Torrifluvents $>$ Fine loamy, mixed, thermic, Typic Haplocalcids (Fluviolacustrine) > Siliceous, thermic, Oxyaquic Torripsamments (Coastal plain) > Sandy, mixed, thermic Typic Haplocalcids - Typic Haplogypsids (old alluvial) > Siliceous, thermic Typic Torripsamments (windblown ). 


\section{Refferences}

Abd El Wahid, M. A. (1976) Status of micronutrients (zinc) in Egyptian soils. ph.D. Thesis. Fac. of Agric. Cairo Univ.

Al-Sharif A.A. (1994) The pedogenic of the calcic formations in Fayoum Depression. Ph.D. Thesis in Cairo Univ.

Al-Sharif, A.A., Soliman, M.M., Riad, M.M., and Abd Elkadar, G.A. (2013) The relation between the geomorphic units and the formed soil characteristics in western Delta Egypt. Egyptian J. of Soil Sciences (in Press).

Badawi, M.A. (1999) Study on soils of central geomorphic units, west of the Nile Delta and their land suitability.

Ball. J. (1939) Contribution to the Geography of Egypt. Cairo Univ.

El-Demerdashe, S.E. (1970) Studies on the nature of inter-ferance between the alluvial and desert soils on the western border of the Nile Delta. Ph.D. Thesis, Fac. of Agric. Cairo Univ.

El-Sayad, E.A. (1983) Studies on some micronutrients in some soils in Fayoum Governorate. M.Sc. Thesis, Fac. Agric., Cairo Univ.

El-Tokhy, M.M. (1987) Studies on the status of some nutrient elements in the soils adjacent to idko lake. M.Sc. Thesis. Fac. of Agric . Cairo Univ.

FAO (1976) A Framework for Land Evaluation. Soil Bull. No. 32, Rome, Italy.

FAO (2010) Guidelines for Soil Description. Food and Agricultural Organization of UNA, Rome.

Fiskell, J.A. (1965) In: Methods of Soil Analysis (part I), Black ,C.A. (Ed.).

Grosuch, T.T. (1959) Radiochemical investigations on the recovery for analysis of trace elements in organic, Analyst, 87, 135.

Hegazy, M.N.A. (1980) Studies on iron availability in soils. M.Sc. Thesis, Fac. Agric. Cairo Univ.

Lindsay, W.L. and Norvell,W.A. (1978) Development of DTPA test for Fe, $\mathrm{Zn}, \mathrm{Mn}, \mathrm{Cu}$ and Cd. Soil Sci., Ame. Proc. 421-428.

McKenzie, R.M. (1959) Aust. J. Soil, 10(1), 52-7.

Munsell color (1975) Munsell color Maceth division of kollnorgen corporation, Maryland, USA.

Oertel, A.C. and Giles, J.B. (1967) Aust. J. Soil Res., 5, 133-47. 
Page, A.L., Miller, R. H. and Keeney, D. R. (1982) Methods of Soil Analysis. part 2. Chemical and Microbiological Properties, $2^{\text {nd }}$ ed., Amer. Soc. of Agron., Madison. Wisconsin, USA.

Rashad, I. F. (1986) Accumulation and distribution of trce elements in soil profiles of ElGabal El- Asfar through long term irrigation with waste water. Ph.D. Thesis, Fac. Agric., Mansoura Univ.

Salam, S.A. (1981) Status of some micronutrients (copper) in Egyptian soils. $P h D$. Thesis, Fac. of Agric. Cairo Univ.

Sandford, K. and Arkell, W. (1939) Palelithic man and Nile valley lower Egypt (with some part of The Red Sea littoral). Univ. of Chicago Orient Inst. Publ vol. XLV.

Soil Survey Staff (2010) Keys to Soil Taxonomy, Eleventh Edition USDA.

Taha, S.A. (1980) Studied on manganese in some soils of Egypt. Ph.D. Thesis. Fac. of Agric. Cairo Univ.

Vinogradov (1959) Geochemistry of Rare and Dispersed Chemical Elements in Soils. (Translated from Russian) Chapman \& Hall Ltd- London.

(Received 31/7/2013;

accepted 20/9/2015) 


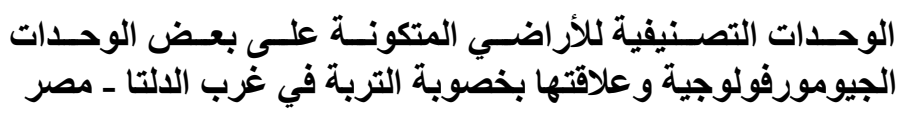

خالد الحدق ، ابراهيم عبد المنعم حجاب ، ايهاب مسعود وجمال فريد

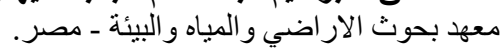

لإجراء هذه الدراسة اختير احد عثر عقد قطاعا ارضيا تمثل الوحدات

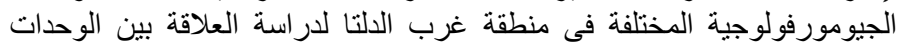

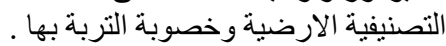

Typic Calcigypsids, أوضحت هذه الدراسة أن الوحدات التصنيفية

Typic تكونت على الأر اضى الرسوبية القديمة . وووحدة Typic Haplocalcid

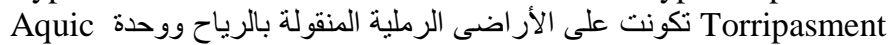

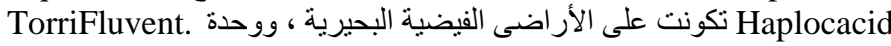
Typic Haplotorrerts Halic Endoaquert , Aridic Endoaquert البحرية ، ووحدة Oxaquic Torripasmment تكونت على أر اضى الثاطىى

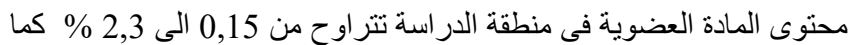

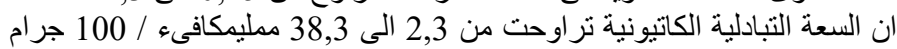

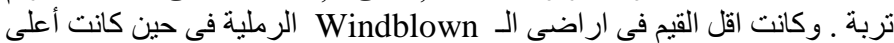

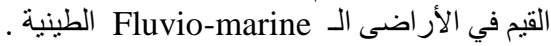

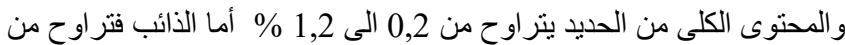

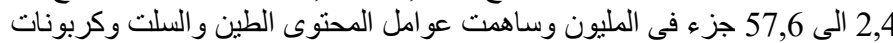
الكالسيوم من 37,1 الى 89,7 من التغير ات في المحتوى الكلى من الحديد .

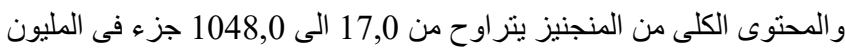

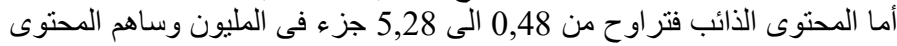
الطين والسلت وكربونات الكالسيوم من 22,7 الى الى الى

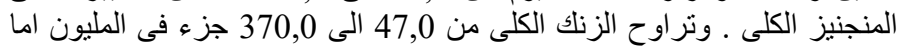

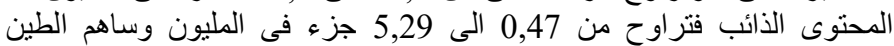

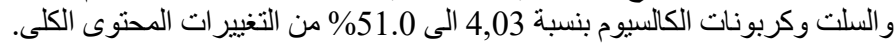

والمحتوى الكلى من النحاس يتراوح من 22 الى الى 168 جزء 169 هزء في المليون

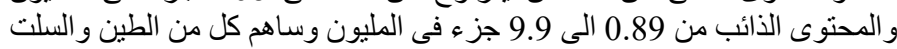
وكربونات الكالسيوم بنسبة 10.9 الى 90.0 \% من التغييرات في في المحتوى الكلى

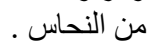

الوحدات التقسيمية المدروسة Typic Haplotorrerts, Endaquerts تكونت على الـ Flood plain, Fluvio marine كانت هي الأعلى خصوبة بية بينما الـ Typic Torripsaments هي الأقل خصوبة . 